\title{
Hydrodynamical simulations of Pinwheel nebula WR 104
}

\author{
Astrid Lamberts ${ }^{1}$, Sebastien Fromang ${ }^{2}$ and Guillaume Dubus ${ }^{1}$ \\ ${ }^{1}$ Laboratoire d'Astrophysique de Grenoble, UMR 5571 CNRS, Université Joseph Fourier, BP \\ 53, 38041 Grenoble, France, email: astrid.lamberts@obs.ujf-grenoble.fr \\ ${ }^{2}$ Laboratoire AIM, CEA/DSM-CNRS-Université Paris Diderot, IRFU/Service d'Astrophysique \\ CEA-Saclay F-91191 Gif-sur-Yvette, France
}

\begin{abstract}
Pinwheel Nebulae are colliding wind binaries (CWB) composed of a Wolf-Rayet star and an early-type star. We first compare our simulations to analytic solutions for CWB. Then we perform large scale 2D simulations of the particular system WR 104. We determine the properties of the gas in the winds and confirm the flow in the spiral has a ballistic motion.
\end{abstract}

Keywords. hydrodynamics, methods: numerical, stars: individual (WR 104), binaries, stars: winds, outflows

\section{Introduction}

Massive stars possess highly supersonic line-driven winds. In binary systems, the interaction of two winds creates a double-shocked structure. For each wind there is a free unshocked component upstream of the shock and a dense, hot shocked wind downstream. The winds are separated by a contact discontinuity (CD). Their momentum flux ratio is given by $\eta=\frac{\dot{M}_{1} v_{\infty 1}}{\dot{M}_{2} v_{\infty 2}}$. The subscripts 1 and 2 respectively stand for the stronger and weaker wind. For $\eta \gg 1$ the weaker wind is collimated. The whole structure looks like a cometary tail and is turned into a spiral due to orbital motion. As a prototype of CWB, we consider WR 104, composed of an O-B star and a WC9 star. The O-B wind is collimated into a very narrow spiral and shows a clear example of Pinwheel Nebula in infrared (Tuthill et al. 2008). The infrared emission is due to the presence of dust in the WR wind, whose origin is still poorly constrained. Up to now simple models of the dust emission in WR 104 have assumed ballistic motion of the shocked winds along an Archimedian spiral (Harries et al. 2004).

\section{Comparison to analytic models}

We use the code hydro RAMSES (Teyssier 2002) with Adaptive Mesh Refinement which enables us to locally increase the spatial resolution. We properly resolve the shock formation and determine the large scale structure at reasonable computational cost. We compared our 2D and 3D adiabatic and isothermal simulations to the solutions of Canto et al. (1996) and Antokhin et al. (2004) with no orbital motion. The 2D results are shown in figure 1 . We show the density map and overplot the analytic solutions.

In the adiabatic case one can clearly distinguish the two shocks and the position of the CD is approximately matched by the analytic solutions. In the isothermal case, the structure is dominated by the 'thin shell instabilities' (Pittard 2009). This cannot be modelled with the analytic solutions. Moreover, the analytic solutions do not account for the orbital motion and for the effect of thermal pressure. These effects can be properly modelled using hydrodynamical simulations. 

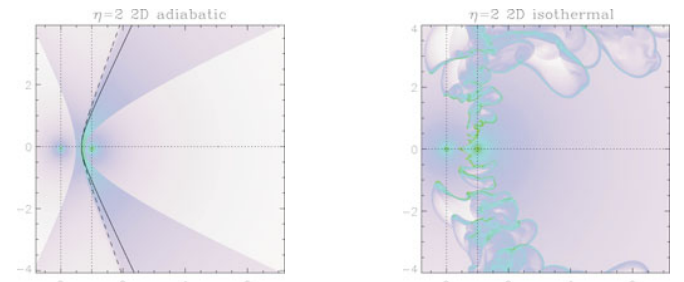

Figure 1. 2D density maps. The stars are located at the intersections of the dotted lines. The dashed line represents the solution from Canto et al. (1996), the solid line the solution from Antokhin et al. (2004). The length scale is given the binary separation.
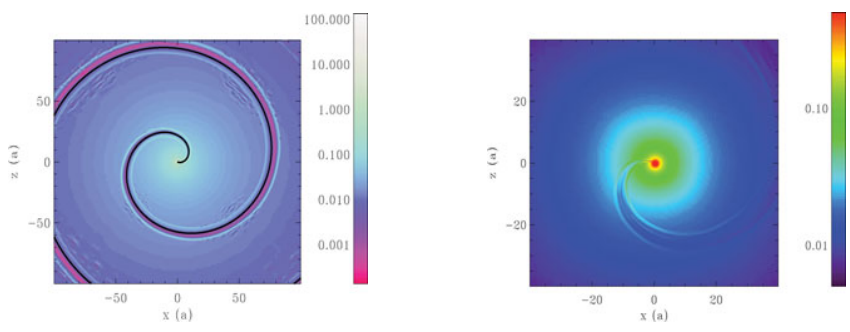

Figure 2. Density in WR 104, adiabatic (left panel) and isothermal (right panel) case.

\section{The case of WR 104}

We made a 2D simulation of WR 104 with adiabatic winds. As $\eta=305$ in WR 104, very high resolution is required to resolve the shocks. The density map is shown in figure 2 on the left panel. The theoretical Archimedean spiral (solid line) matches the results of the simulation. Different components of the wind can be seen. Most of the gas is the free WR wind. The densest zone is the shocked WR wind at the edges of the spiral. The density in the shocked O-B wind in the spiral appears to be steady. The velocity is constant in the spiral, confirming ballistic motion.

Cooling is important in the WR wind, suggesting an isothermal equation of state is more appropriate. We thus expect the presence of thin shell instabilities, which might help mixing. We made a $2 \mathrm{D}$ simulation with an isothermal equation of state, as is shown on the right panel of fig. 2. No instabilities can be seen. The extreme confinement of the O-B star wind could prevent their developpement. This could also be due to insuficient numerical resolution

\section{Summary and conclusions}

We performed 2D large scale simulations of WR 104, completely modelling one step of the spiral structure. This work confirms ballistic motion along Archimedean spiral. More analysis is necessary to put stronger constrains on dust formation. Further work will focus on the phenomena triggering or preventing the thin shell instabilities.

\section{References}

Antokhin, I. I., Owocki, S. P. \& Brown, J. C. 2004, ApJ, 611, 434

Canto, J., Raga, A. C. \& Wilkin, F. P. 1996, ApJ, 469, 729

Harries, T. J., Monnier, J. D., Symington, N. H., \& Kurosawa, R. 2004, MNRAS, 350, 565

Pittard, J. M. 2009, MNRAS, 396, 1743

Teyssier, R. 2002, A\&AA, 385, 337

Tuthill, P. G., Monnier, J. D., Lawrance, N., Danchi, W. C. et al. 2008, ApJ, 675, 698 